\title{
Using Charge-Coupled Devices to Characterize Neutron Events
}

\section{Dylan Smith - Department of Physics and Astronomy, Michigan State University Erik Ramberg - Fermi National Accelerator Laboratory}

\section{Introduction}

- Ultra-cold neutrons (UCNs) < 300 neV of kinetic energy

- Useful for studying position-dependent quantum states, as they are only influenced by Earth's gravity

- Precise measurements involving dark matter, dark energy, and quantum gravity

- Detecting neutrons involves the reaction in a coated CCD of

$$
{ }^{10} B+n \rightarrow{ }^{7} L i+\alpha+\text { free energy }
$$

- A simulation software package called The Stopping and Range of lons in Matter (SRIM) uses Monte Carlo methods to track ions in a chosen target
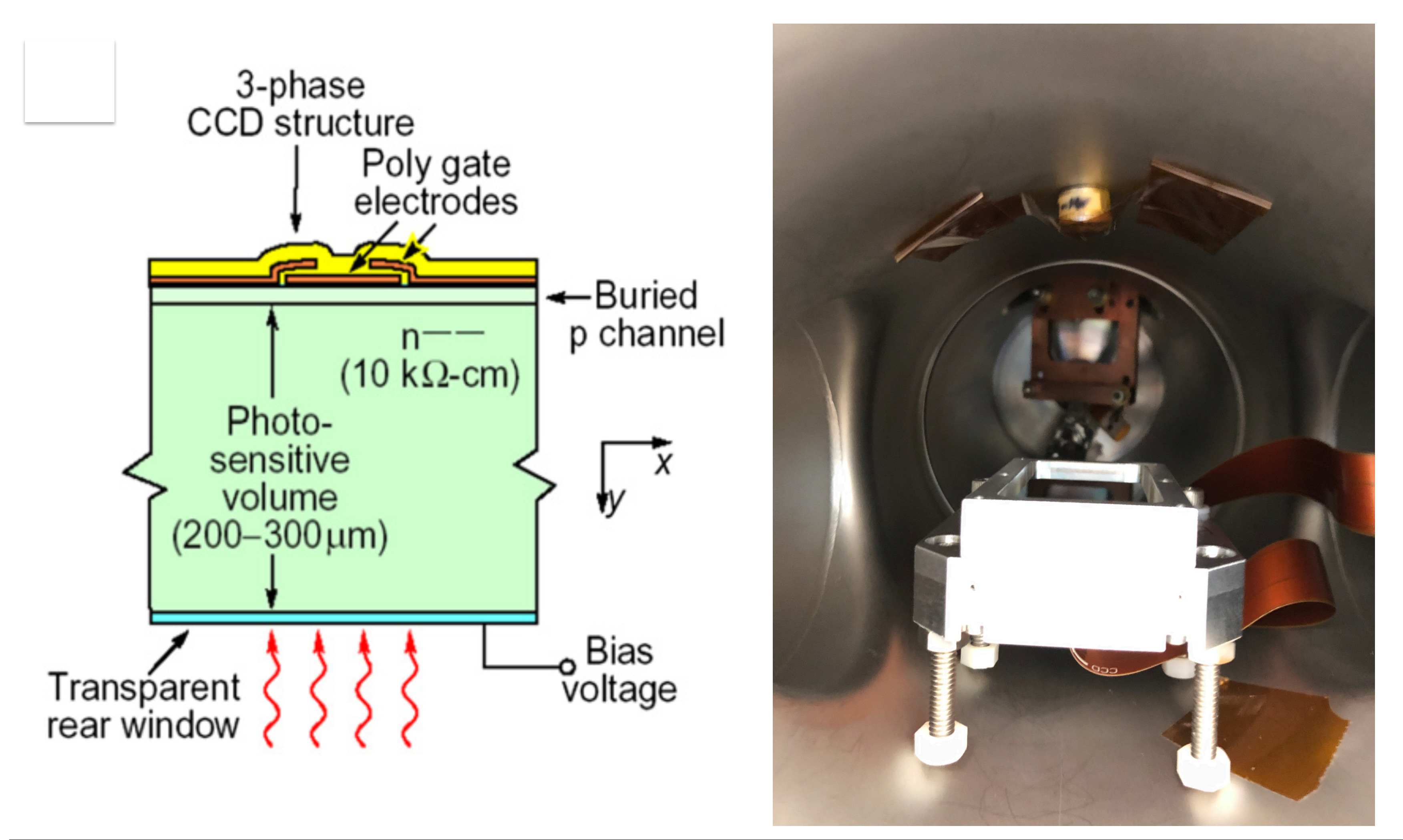

Fig. 1: Diagram of Boroncoated CCD design (left) and picture of CCD set-up (right)

\section{Methods}

- Performed calibrations/measurements on uncoated CCD

- The purpose of this is to ensure its viability and send it to Los Alamos National Laboratory to be coated in ${ }^{10} \mathrm{~B}$

- CCD is placed in a $10^{-4}$ Torr vacuum chamber at $140 \mathrm{~K}$

- The CCD is connected to a Low Threshold Acquisition (LTA) device, which converts the CCD hits into an image file

- The CCD was characterized by ${ }^{55} \mathrm{Fe}$ and ${ }^{252} \mathrm{Cf}$ sources - ${ }^{55} \mathrm{Fe}$ is used to determine the gain and noise of the CCD - ${ }^{252} \mathrm{Cf}$ is used to measure penetrating hits on the CCD

- SRIM is used to determine a correlation between incident ion angle and 2D variances

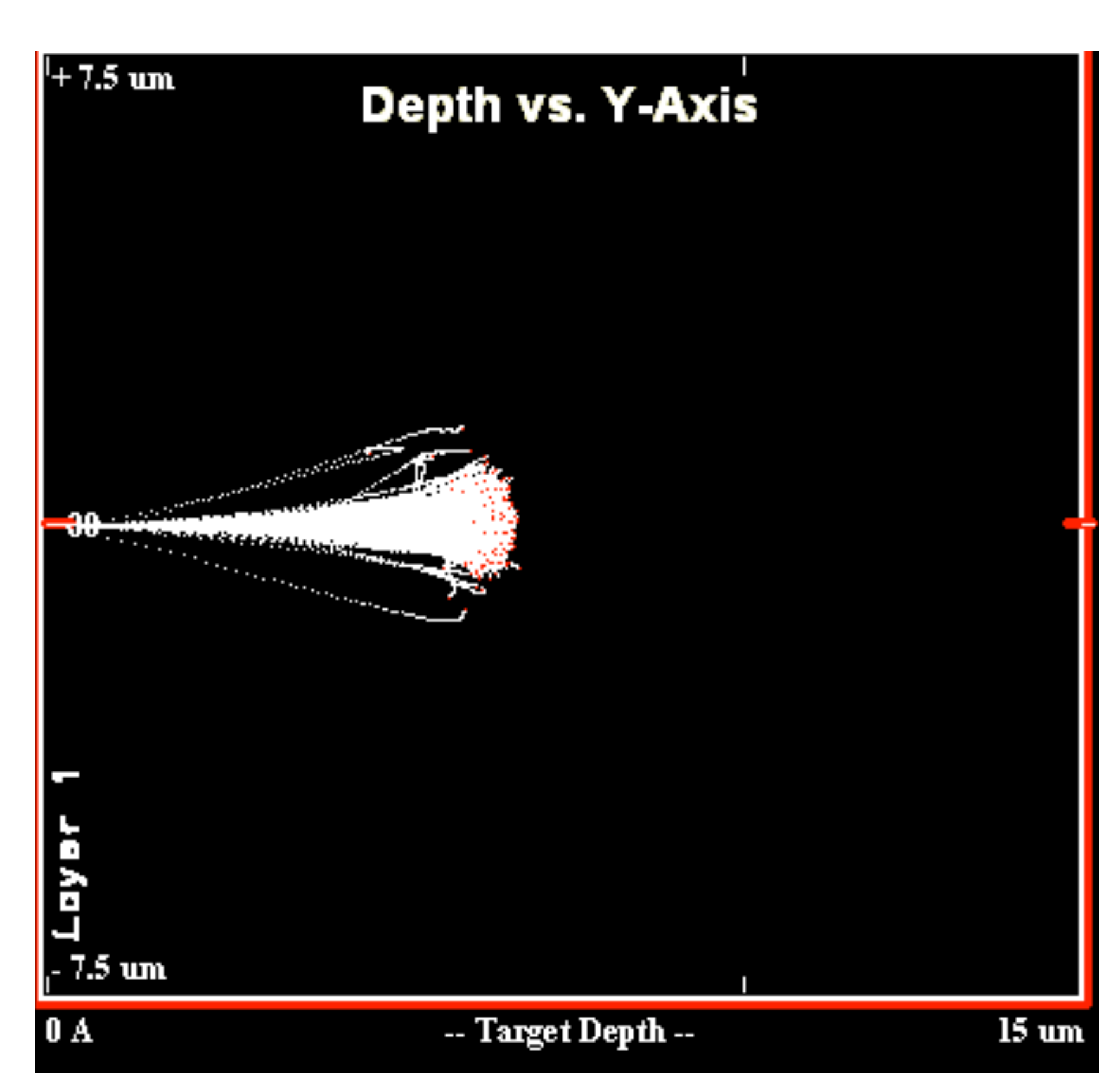

Fig. 2: SRIM output of $\alpha$ particle hitting a silicon target at $1.7 \mathrm{MeV} .1000$ tracks are shown

\section{Results}

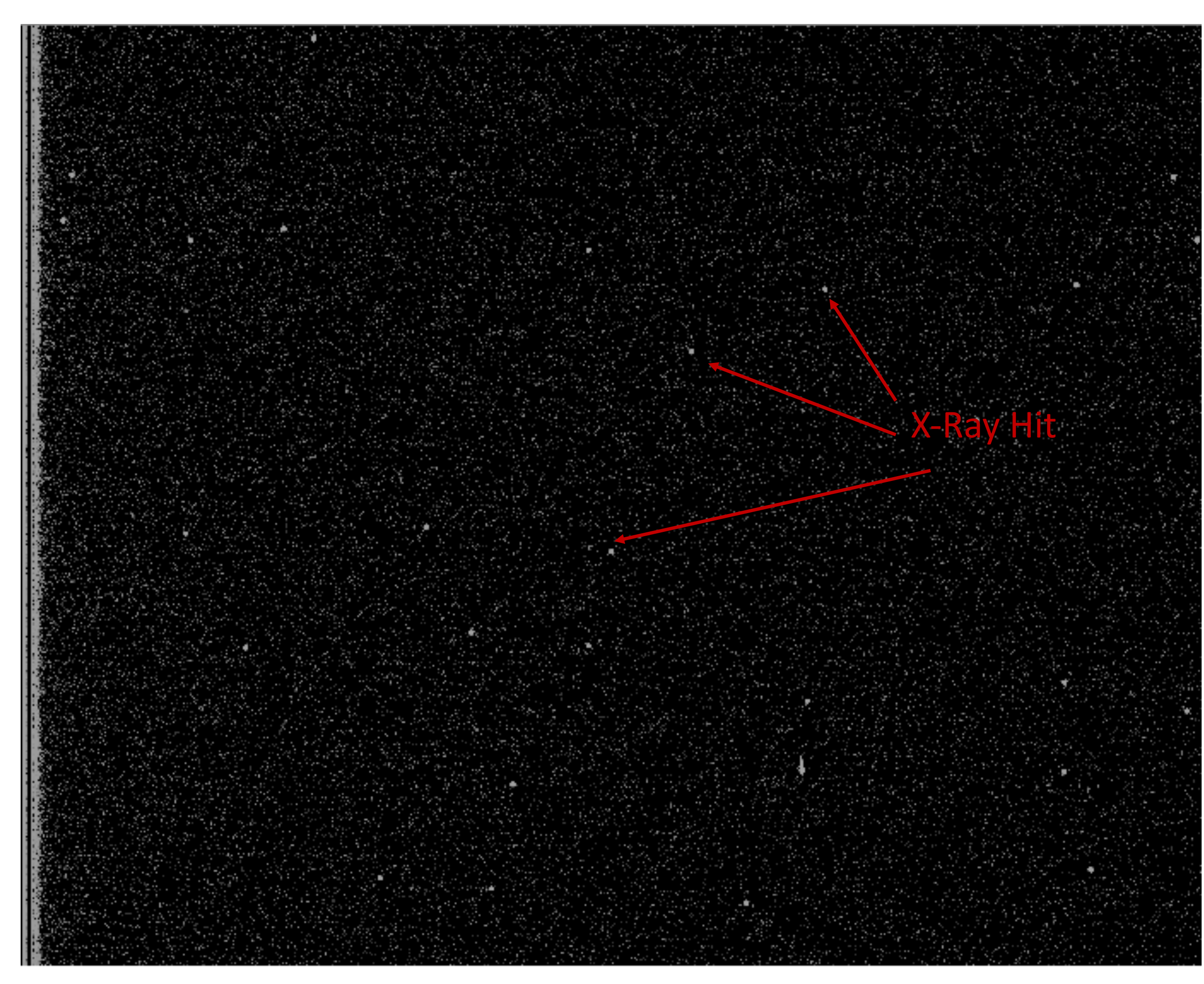

Fig. 3: CCD image with an ${ }^{55} \mathrm{Fe}$ source. The slightlybrighter dots are the $\mathrm{x}$-ray events

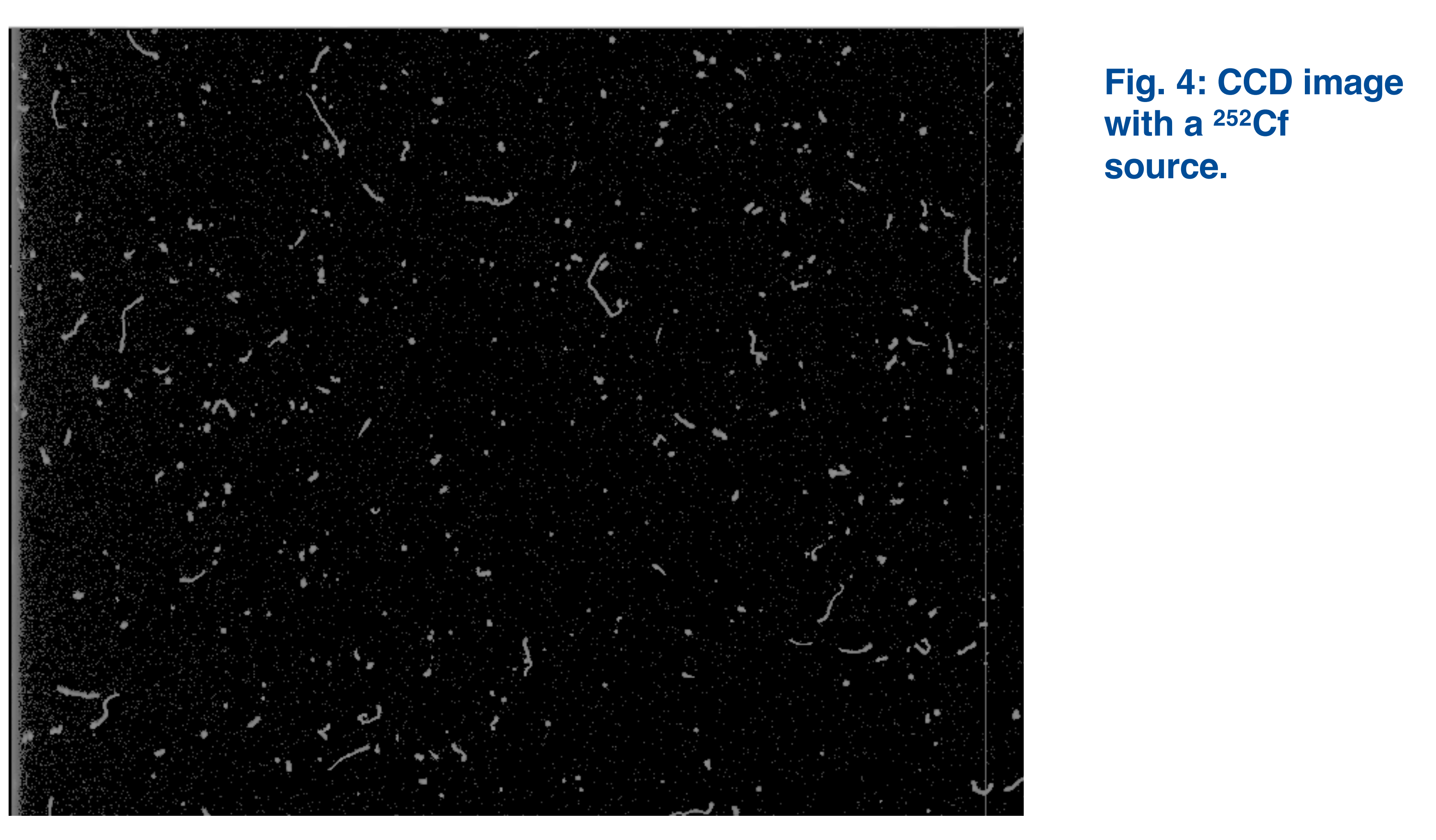

\section{Discussion/Conclusions}

- Values calculated from ${ }^{55} \mathrm{Fe}:$

- Gain = 48.2 ADU/e-

- Noise $=11.5 \mathrm{e}^{-}$

- Large amount of hits with ${ }^{252} \mathrm{Cf}$ source shows that the CCD is working properly

- Still working on finding correlation between variance and angle in SRIM

\section{Acknowledgements}

This manuscript has been authored by Fermi Research Alliance, LLC under Contract No. DE-AC02-07CH11359 with the U.S. Department of Energy, Office of Science, Office of High Energy Physics.

\section{References}

E. Ramberg, J. Estrada, V. Nesvizhevsky, High Position Resolution Imager for Mapping Gravitational Quantum States using Ultra Cold Neutrons, DOE HEP National Laboratory Announcement Number: LAB 19-2077 